\title{
sciendo DETERMINING AND ANALYSING SUPPORT CONDITIONS AT VARIABLE CONSTRUCTION OF CRANKSHAFTS
}

DOI 10.2478/ntpe-2018-0069

\author{
dr hab. inż. Krzysztof Nozdrzykowski, Prof. AM. \\ dr inż. Zenon Grządziel \\ Maritime University of Szczecin, Poland \\ Ing. Jozef Harušinec, PhD \\ University of Zilina, Slovak Republic
}

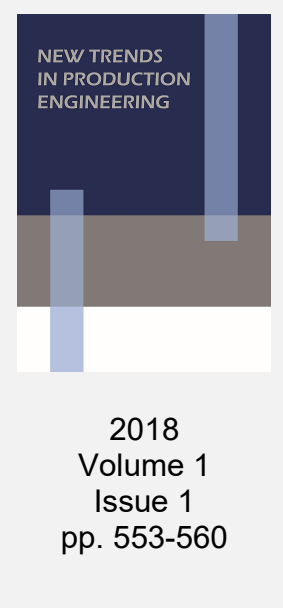

\begin{abstract}
The article presents study results of the influence of crankshaft construction changes on the choice of support conditions allowing to eliminate deflections and elastic deformations of crankshafts under their self-weight. For the purpose of this study we implemented a programme for strength calculations Nastran FX 2010 which enables modelling the research subject with a finite element method and counting the value if reaction forces ensuring zero value of deflections on main journals at a change in the crankshaft's angle of rotation.
\end{abstract}

Keywords: crankshafts, support conditions, deflections and elastic deformations

\section{INTRODUCTION}

Crankshafts of the ship engines are the elements of big mass and dimensions, but at the same time they are limp elements of low and variable stiffness, prone to bending deformations. Due to their properties such shafts require ensuring support conditions which would guarantee elimination of their elastic deformations under their self-weight (Dai, S.P. et. al. 2006, Sun, J. et. al. 2010, Quan, W. et al., 2011, Walczak, W. et al., 2011). The issue of determining support conditions which would guarantee elimination of shaft's elastic deformations is of great importance, especially in case of estimation of geometrical condition of crankshafts realized at the stage of their production or repairs and regeneration. Uncontrolled support conditions generate errors which distort measurements of geometrical quantities, especially deviations of shape and location of crankpin and journal axles. The majority of measurements require turning the shaft during the measurement itself. In such a case the possibilities of supporting shafts are significantly limited and they depend on shafts' construction so, among others, on the number and distribution of its crankpins. Construction changes of the shafts directly influence the value of reaction forces occurring at the joints of supports' heads of the locking elements supporting a shaft during measurements.

A range of studies conducted so far have shown that for the total elimination of deflections and elastic deformations of the shafts it is necessary that the values of reaction forces occurring at the joints of the relieving supports' heads changed not only at the length of the shaft, but also, depending on the shaft's rotation angle, on the supports (Jadhav, A. et. al. 2013, Fonte, M. et. al. 2015, Nozdrzykowski, K. 2015, Nozdrzykowski, K. and Grządziel, Z. 2015). Construction details of crankshafts for the engines of similar parameters (low-, medium- and high-speed engines) may cause the differences between the engines both with respect to shape and mutual location of the cranks, and size what, in turn, influences the mass and weight of the shaft. Needless to say that taking into account a change of mass and weight of the shaft, the material it is made of and the type of surface treatment, play an important role (Chybowski, L. and Gawdzińska, K. 2016).

This article presents the analysis results of the influence of construction changes on the choice of support conditions which guarantee the elimination of deflections and elastic deformations of crankshafts. 


\section{ANALYSIS OF SUPPORT CONDITIONS OF CRANKSHAFTS OF VARIABLE CONSTRUCTION}

The analysis involved constructional changes in crankshaft of eight-cylinder, medium-speed engine of the main drive of the ship Buckau Wolf R8DV 136. The crankshaft's mass was 9360 $\mathrm{N}$ and its length $3600 \mathrm{~mm}$; the diameter of main journals was $149 \mathrm{~mm}$ and crankpins $144 \mathrm{~mm}$. Crank webs are oval and have dimensions $252 \times 358 \mathrm{~mm}$ (Fig. 1). The changes concerned dimensions and distribution of journals and crankpins, as well as shape and dimensions of crank webs and their mutual location. For the purpose of this study we used a programme for strength calculations Nastran FX 2010 which enables modelling the research subject with a finite element method and counting the value if reaction forces ensuring zero value of deflections on main journals at a change in the angle of rotation of crankshafts which differ from each other in construction details.

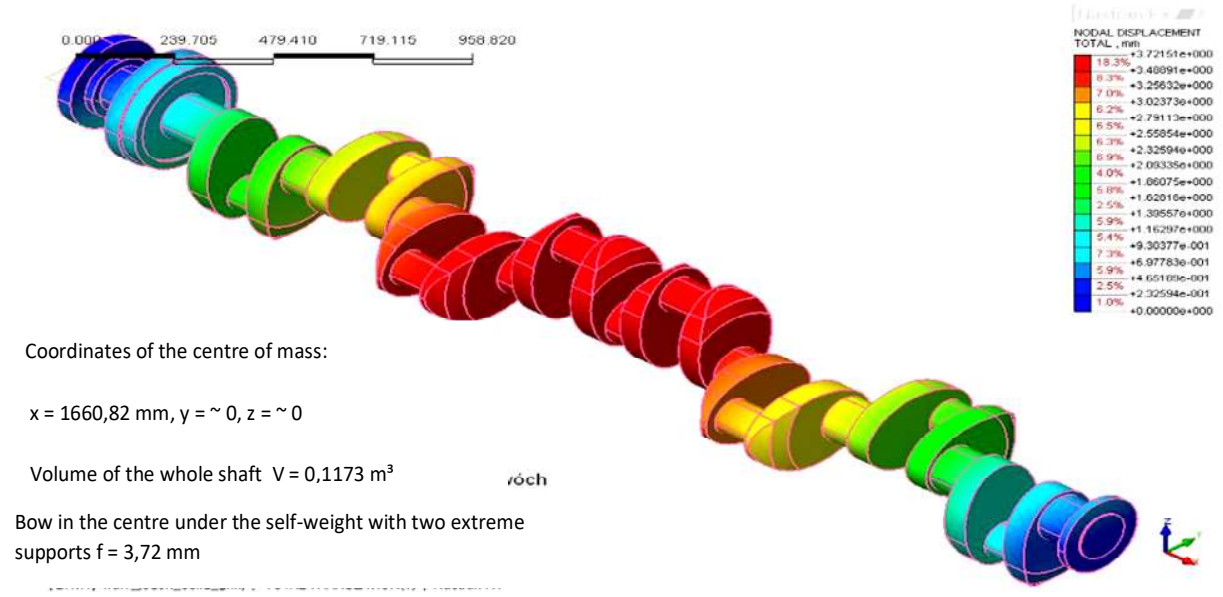

Fig. 1. Crankshaft of eight-cylinder, medium-speed engine of the main drive of the ship Buckau Wolf R8DV 136.

Example study results have been presented in a form of diagrams presented in polar and Cartesian coordinate system

Fig. 2 present distribution of forces ensuring zero value of deflections on main journals for the original crankshaft of construction and dimensions in line with its execution drawing with the diameter of main journals being $149 \mathrm{~mm}$ and crankpins - $144 \mathrm{~mm}$, and with oval crank webs of dimensions $252 \times 358 \mathrm{~mm}$.
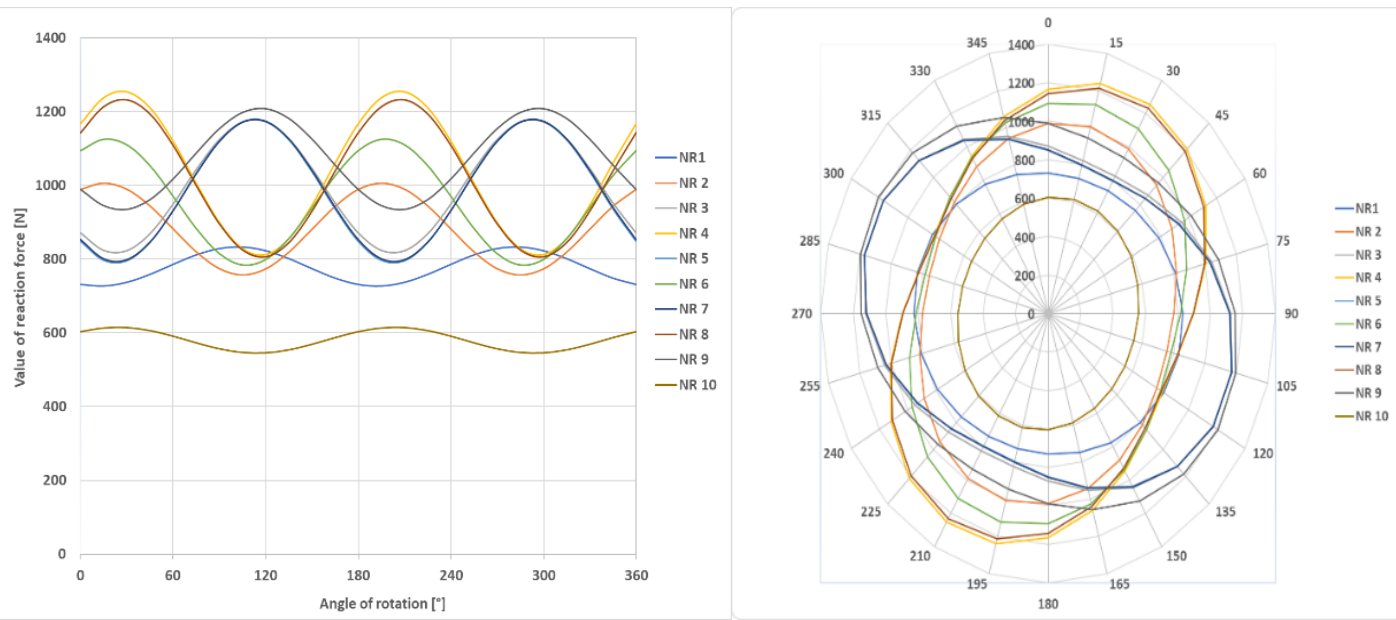

Fig. 2. Distribution of reaction forces that guarantee zero value of deflections on main journals for a crankshaft of diameter of main journals being $149 \mathrm{~mm}$ and crankpins $-144 \mathrm{~mm}$, and with oval crank webs of dimensions $252 \times 358 \mathrm{~mm}$. 
Fig. 3 present a change in distribution of reaction forces that guarantee zero value of deflections on main journals for a crankshaft of diameter of main journals being $149 \mathrm{~mm}$ and reduced diameter of crankpins $-114 \mathrm{~mm}$, and with oval crank webs of dimensions $252 \times 358$ $\mathrm{mm}$.
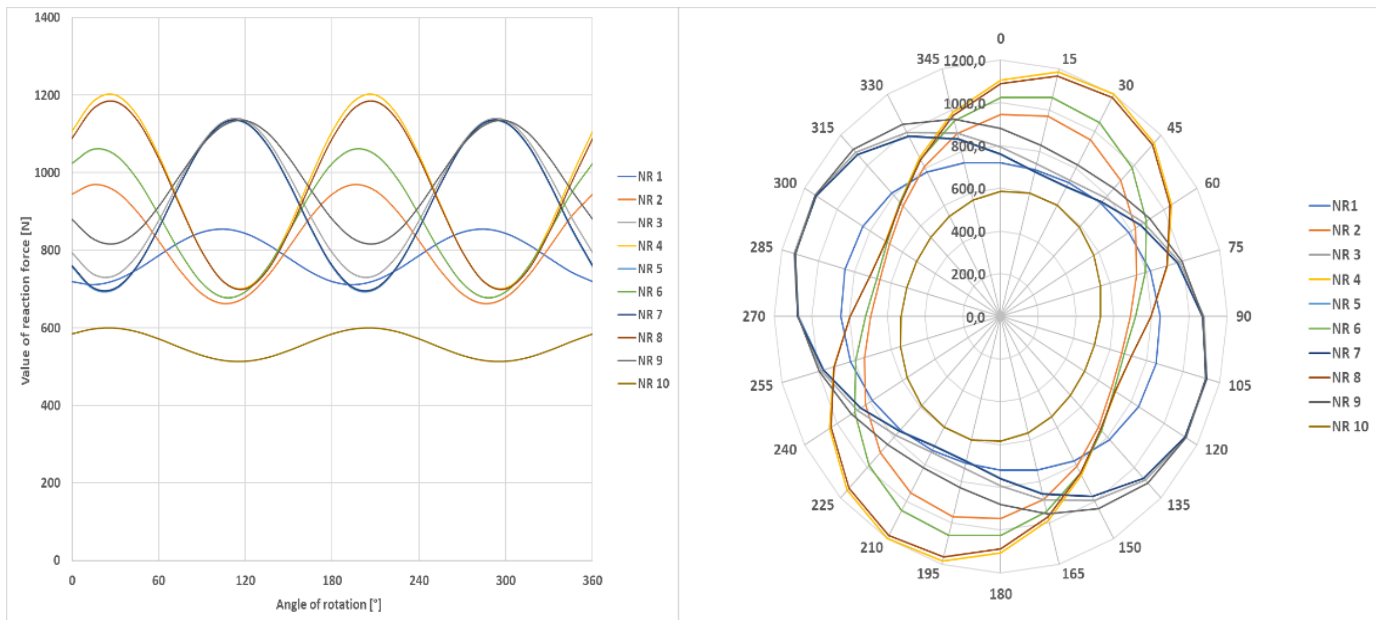

Fig. 3. Distribution of reaction forces that guarantee zero value of deflections on main journals for a crankshaft of diameter of main journals being $149 \mathrm{~mm}$ and crankpins $-114 \mathrm{~mm}$, and with oval crank webs of dimensions $252 \times 358 \mathrm{~mm}$.

Fig. 4 present distribution of reaction forces that guarantee zero value of deflections on main journals for a crankshaft of diameter of journals being $149 \mathrm{~mm}$ and crankpins - $144 \mathrm{~mm}$, but with a change in shape of crank webs which have a circular shape of a diameter 358mm.
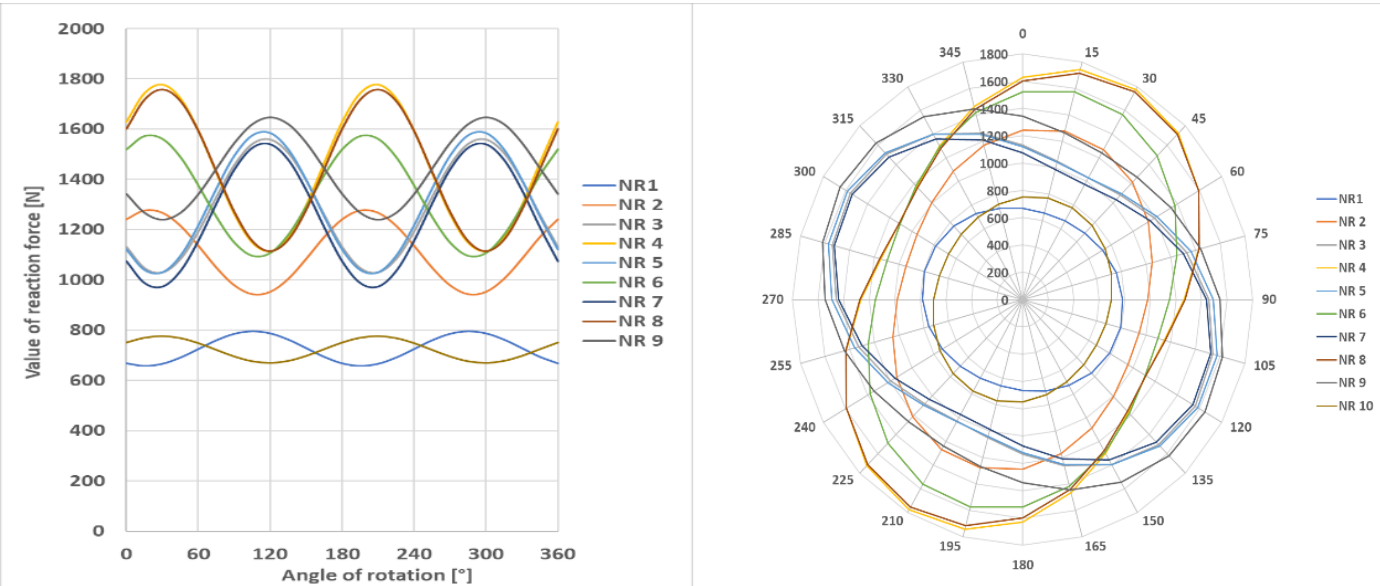

Fig. 4. Distribution of reaction forces that guarantee zero value of deflections on main journals for a crankshaft of diameter of journals being $149 \mathrm{~mm}$ and crankpins - $144 \mathrm{~mm}$, and with circular crank webs of dimension $358 \mathrm{~mm}$.

As it has been shown, the diagrams of reaction forces values depending on rotation angle of the shaft on the particular journals, presented in polar coordinate system take the form of more or less deformed ellipse what, in Cartesian coordinate system corresponds to the graph of function similar to cosine. Such a presentation of distribution of forces for particular journals on one diagram enables visualisation of their mutual location as well as their estimation both in quantitative and qualitative terms.

A change in values of reaction forces of the support depending on the angle of rotation of the shaft is caused by the fact that the surfaces in the consecutive cross sections to the main axle of the shaft have different areas, and their centres of gravity lie in different directions and different distances from the shaft's main axle. Due to this fact, during the rotation of the shaft 
mutual interactions between the masses of subsequent cranks located at various angles to each other occur. These interactions take different nature and form, depending on the shaft construction. As a result, during the rotation of the shaft its particular parts undergo elastic deformations, not only flexural, but also torsional (Zhao, Y. and Cao, S.Q. 2013, Nozdrzykowski, K. 2015).
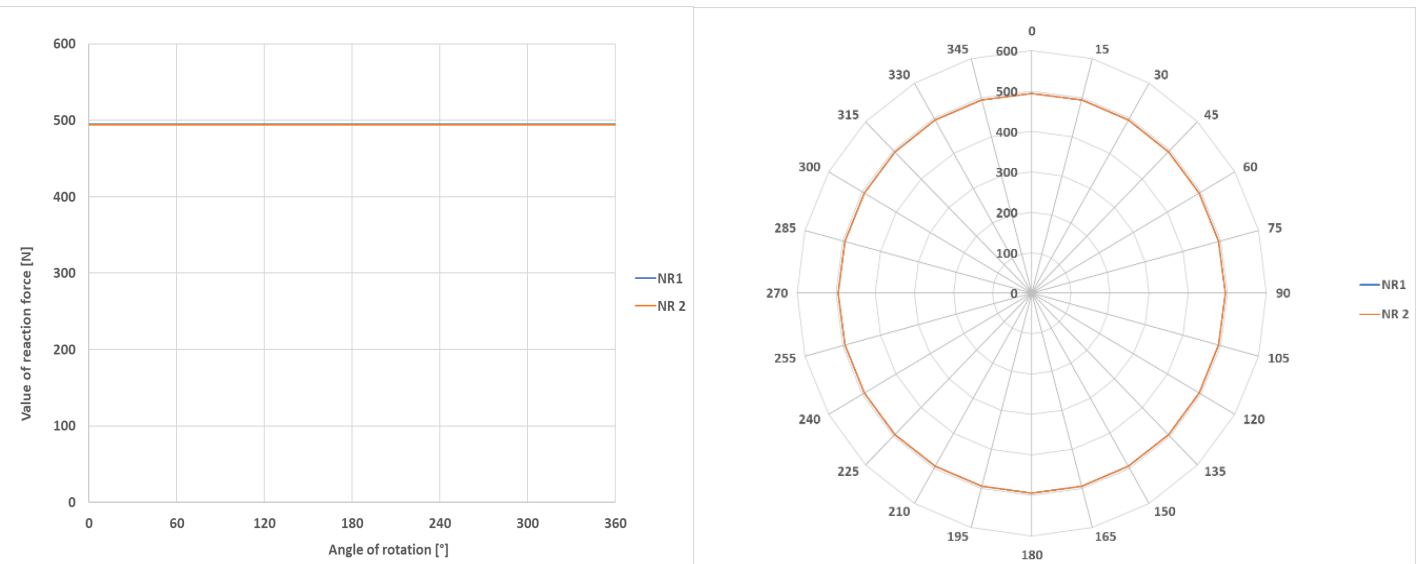

Fig. 5. Distribution of reaction forces that guarantee zero value of deflections on journals for a singular crank.

In case of singular crank, reaction forces on the journals take a constant value regardless of the angle of rotation of the shaft on the supports, what has been shown in Fig. 5. This is a statically determined system and both reaction forces o journals are equal and at the same time they correspond to a half of the external load, regardless of the angle of rotation of the shaft. But in case of two cranks connected to each other the distribution of reaction forces on the journals exhibits the variability of reaction forces on the journals at the change in the angle of rotation of the shaft, what can be seen in Fig. 6 and Fig. 7. The value of reaction forces on the extreme journals are much smaller than reaction force on the middle journal. Distribution of reaction forces that guarantee zero value of deflections on journals for two cranks located in one surface rotated to each other about their axle by the angle of $180^{\circ}$, have been presented in Fig. 6. Fig. 7 show distribution of reaction forces that guarantee zero value of deflections on journals for two cranks located perpendicularly to each other. But for cranks located in one surface, rotated to each other about their axle by the angle of $180^{\circ}$ (Fig. 6), the amplitudes of support reaction forces are much smaller.
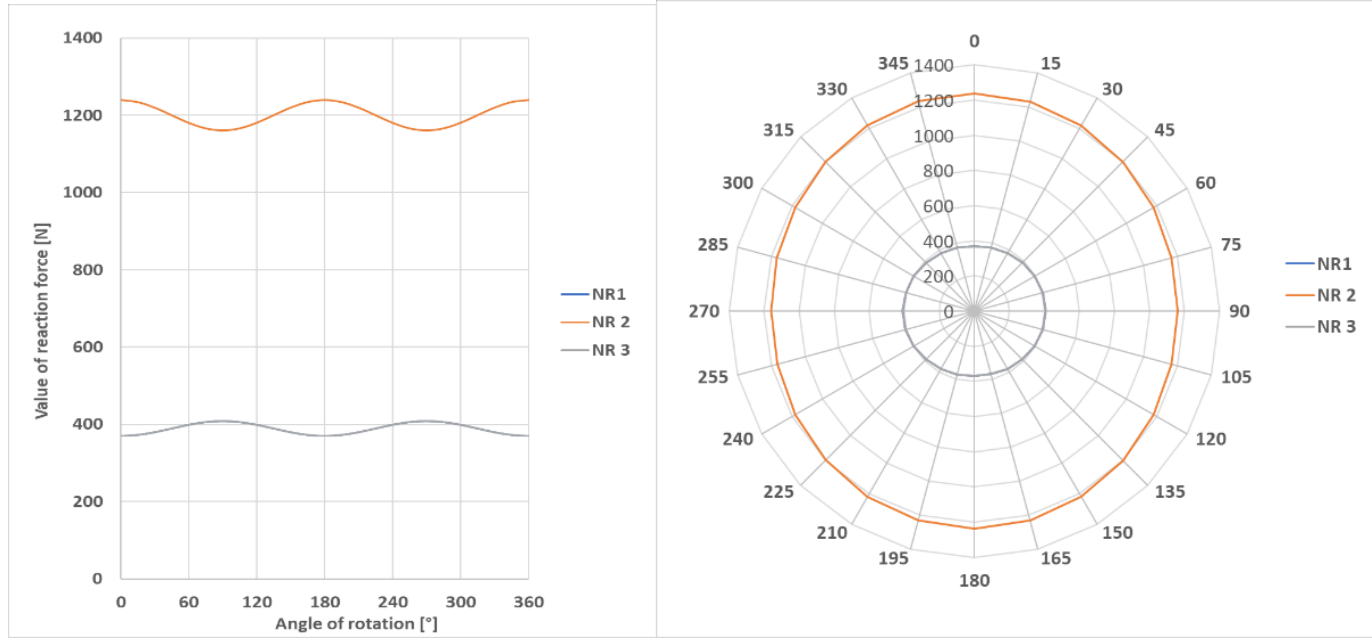

Fig. 6. Distribution of reaction forces that guarantee zero value of deflections on journals for two cranks located in one surface, rotated to each other about their axle by the angle of $180^{\circ}$. 

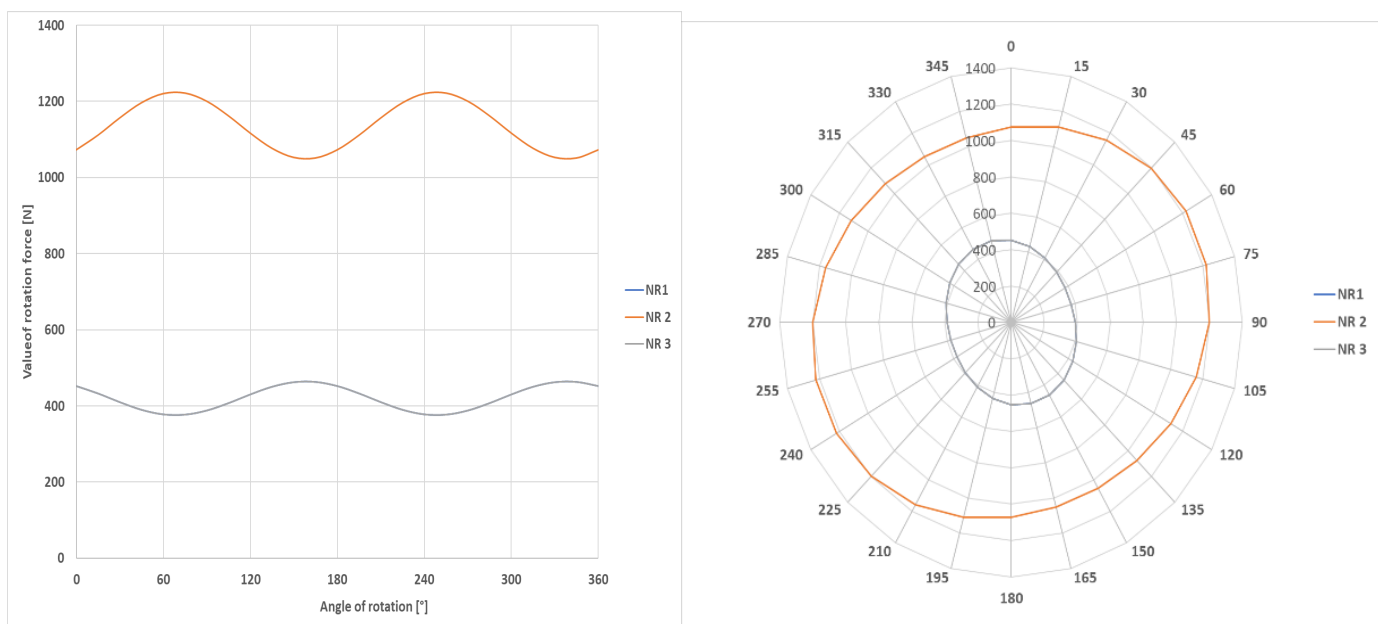

Fig. 7. Distribution of reaction forces that guarantee zero value of deflections on journals for two cranks located perpendicularly to each other

What is more, it is characteristic that regardless of type of changes in the construction of the shaft the diagrams showing the distribution of reaction forces that guarantee zero value of deflections on the particular journals are shifted with relation to each other depending on the angular location of cranks, what has been shown in Fig. 9, 11 and 13. Fig. 9 presents the distribution of reaction forces that guarantee zero value of deflections on journals, where particular cranks are located perpendicularly to each other (Fig. 8).

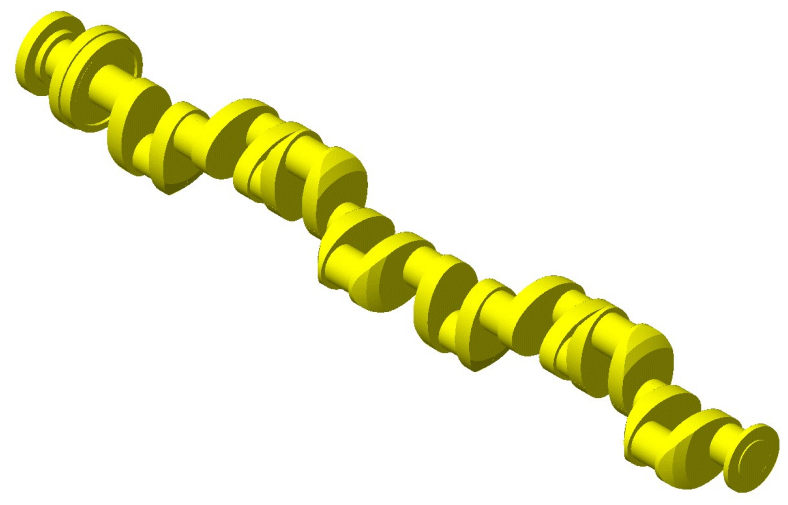

Fig. 8. Shaft whose subsequent cranks are rotated to each other about their axle by the angle of $90^{\circ}$
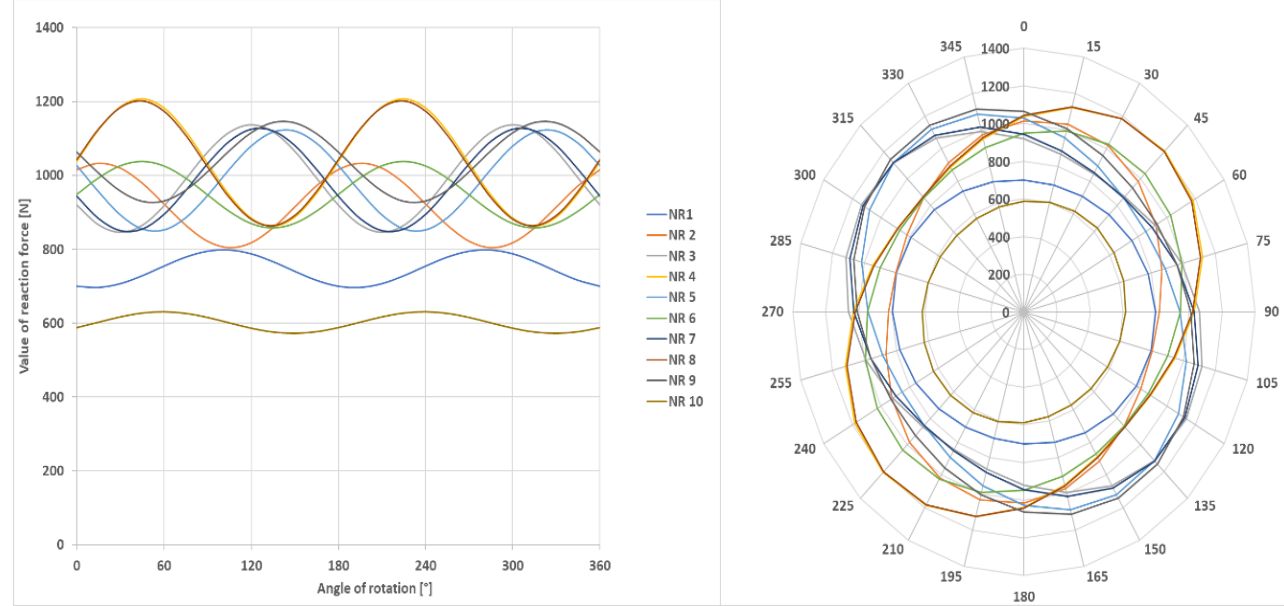

Fig. 9. Distribution of reaction forces that guarantee zero value of deflections on journals when subsequent cranks are rotated to each other about their axle by the angle of $90^{\circ}$. 
Fig. 11 presents the distribution of reaction forces that guarantee zero value of deflections on journals, where particular cranks are rotated to each other about their axle by the angle of $180^{\circ}$ (Fig. 10). But Fig. 13 shows the distribution of reaction forces that guarantee zero value of deflections on journals, where particular cranks are rotated to each other about their axle by the angle of $120^{\circ}$ (Fig. 12). The smallest amplitudes of reaction forces on the supports occur in case when particular cranks are rotated to each other about their axle by the angle of $120^{\circ}$ (Fig. 13), and the biggest when the cranks are rotated to each other about their axle by the angle of $90^{\circ}$ (Fig. 9).

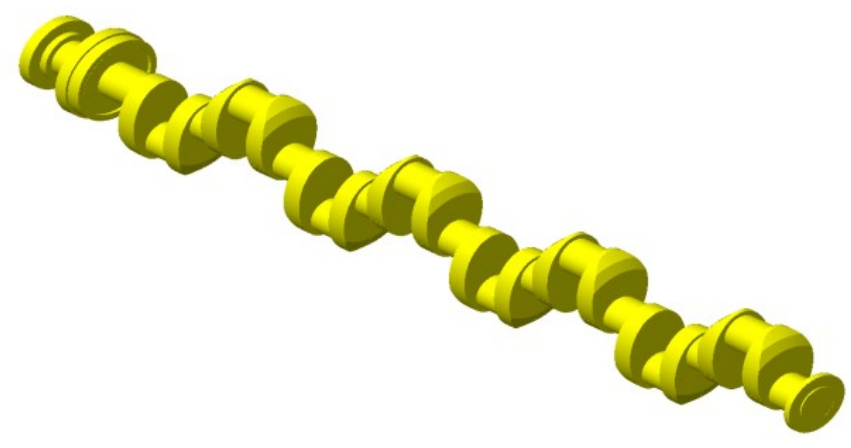

L.

Fig. 10. Shaft whose subsequent cranks are rotated to each other about their axle by the angle of $180^{\circ}$
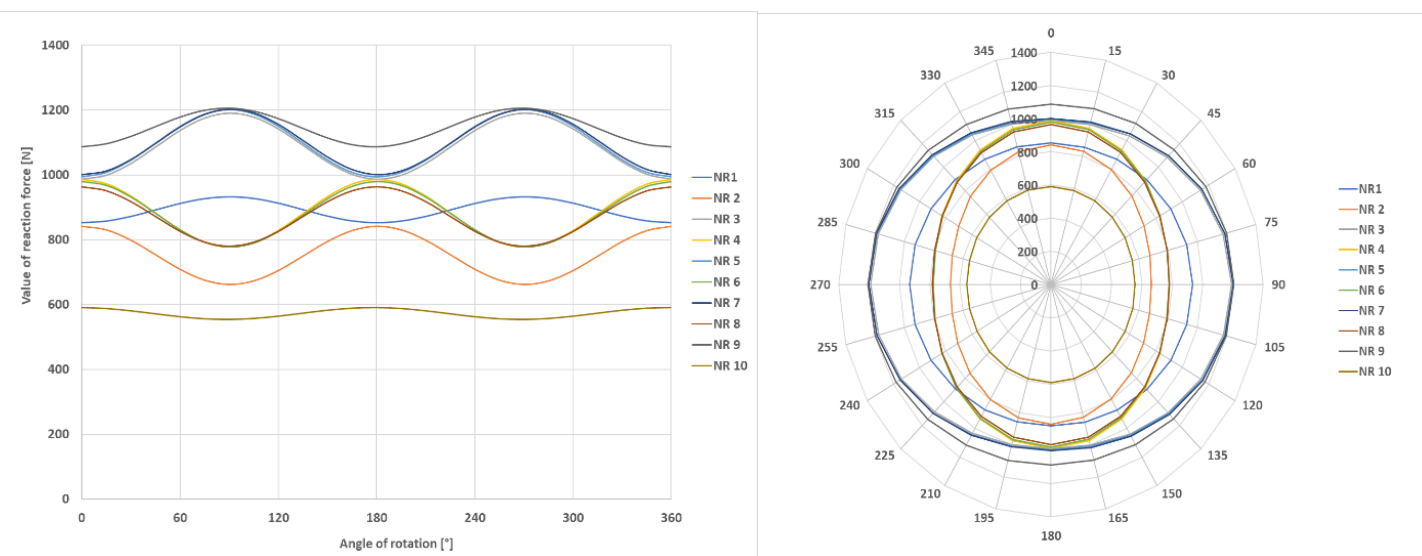

Fig. 11 Distribution of reaction forces that guarantee zero value of deflections on journals when subsequent cranks are rotated to each other about their axle by the angle of $180^{\circ}$.

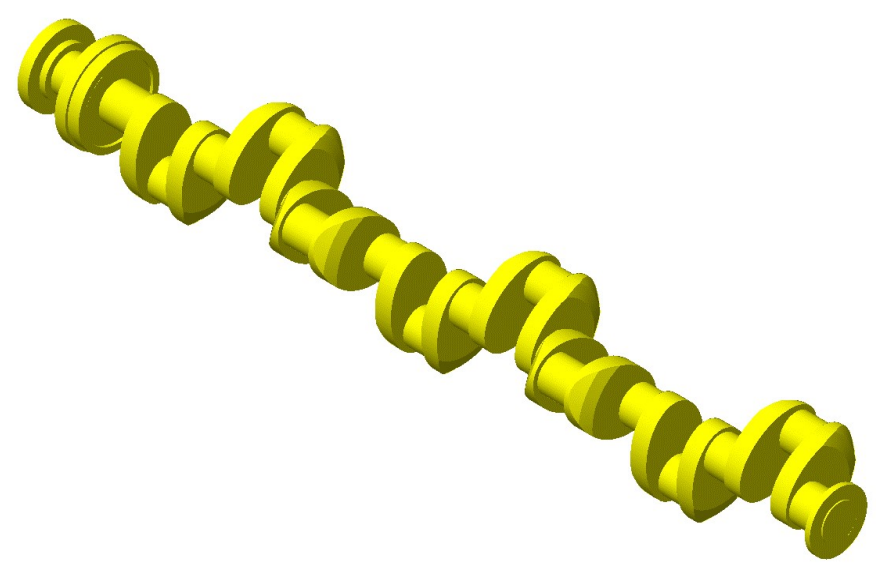

Fig. 12. Shaft whose subsequent cranks are rotated to each other about their axle by the angle of $120^{\circ}$ 

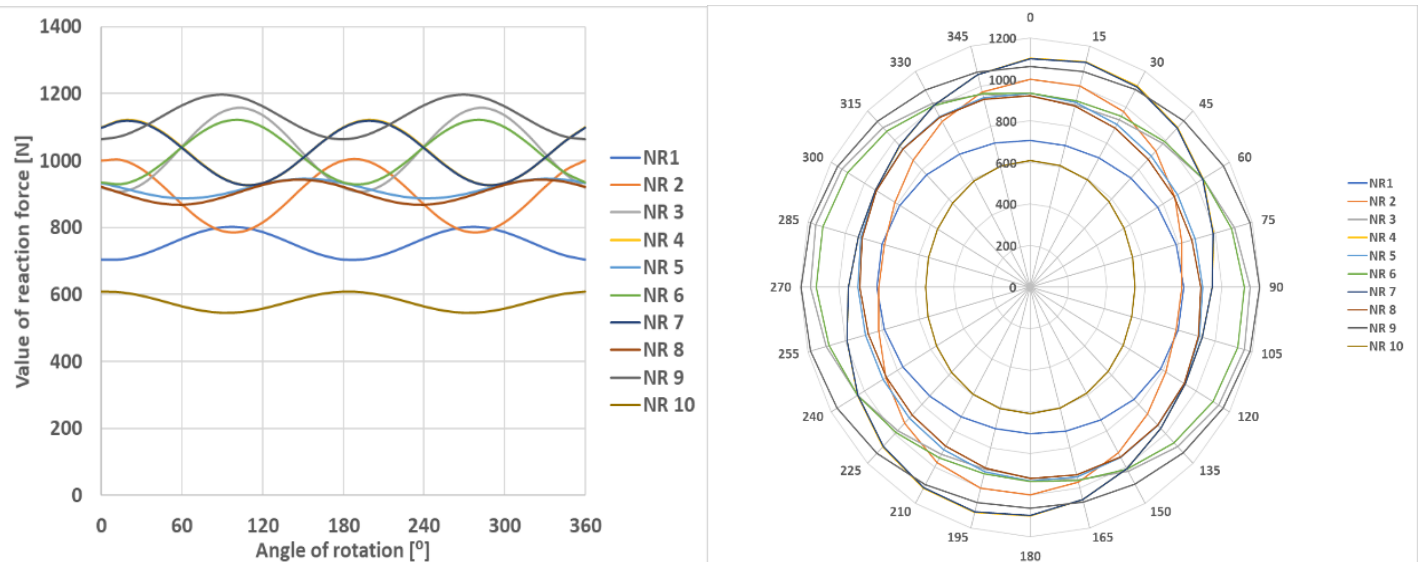

Fig. 13. Distribution of reaction forces that guarantee zero value of deflections on journals when subsequent cranks are rotated to each other about their axle by the angle of $120^{\circ}$.

\section{CONCLUSIONS}

Conducted studies have clearly shown the need for realization of variable values of reaction forces on the particular journals during the measurements of deviations and geometrical quantities of crankshafts. The values of reaction forces should change depending on the angular location of the shaft, according to the results of strength calculations carried out earlier. The selection of forces should be realized individually for a particular type of shaft taking its construction features into account. The studies' results additionally reveal the nature of mutual interaction between particular cranks and the influence of this interaction on the distribution of reaction forces of the supports at the variable construction of the shaft. These results also underpin establishing a universal calculating programme which enables making calculations of the required force values eliminating deflections and elastic deformations of crankshafts of different constructions. This programme finds practical application during measurements of crankshafts' geometrical deviations realized with the use of measuring system with, so called, elastic support of the measured subject and controlled force value of variable interaction of the supports. The type of support used, at the correct selection of lift-off forces calculated before, effectively compensates deflections and elastic deformations of the measured subject under its self-weight, guaranteeing realization accuracy of the geometrical quantities' measurements.

The clue of the studies can also be expressed in a form of statement that the commonly used traditional type of support, namely support of the crankshaft with the system of few fixed, stiff prism supports of a regular height has a limited possibility to eliminate elastic deformations of the shaft due to its low and variable stiffness and, resulting from it, high susceptibility to elastic deformations, both flexural and torsional. Consequently, we accept their functioning during measurements (for instance, the necessity of measurement of so called elasticity of shaft's crank webs) and assume that if the value of elasticity remains within the acceptable limits, then the measurements of geometrical quantities realized in these conditions may be considered satisfactory.

\section{REFERENCES}

Dai, S.P., Gao, L., Gao, K. (2006). The applications of measurement system for crankshaft. 1st International Symposium on Digital Manufacture, vol. 1-3, pp. 833-836.

Fonte, M., Duarte, P., Anes, V., Freitas, M., Reis, L. (2015). On the assessment of fatigue life of marine diesel engine crankshafts. Engineering failure analysis, vol. 56, p. 51-57.

Jadhav, A., Chalwa, V., Gaikwad, P. (2013). Fatigue Failure Analysis Of Engine Crankshaft. International Journal of Engineering Research \& Technology 2, pp. 614-621.

Chybowski, L., Gawdzińska, K. (2016). On the Present State-of-the-Art of a Component Importance Analysis for Complex Technical Systems. Advances in Intelligent Systems and Computing, Volume 445, Springer International Publishing, pp. 691-700.

Nozdrzykowski, K., (2015). Prevention of Elastic Strains in Flexible Large Size Machine Parts with the Use of Elastic Support, Machine Dynamics Research, vol. 39, No 2, pp. 11-122. 
Nozdrzykowski, K., Grządziel, Z. (2015). Simulation tests and measurements of crankshaft deformations by the symmetric method. Scientific journals of the Maritime University of Szczecin, vol. 42, pp. 33-37.

Quan, W., Wei, J., Zhao, Y.W., Sun, L.H., Biani, XY. (2011). Development of an Automatic Optical Measurement System for Engine Crakshaft. 2011 International Conference on Computational Science and Applications, pp. 68-75.

Sun, J., Wang, J., Gui, C. (2010). Whole crakshaft beam-element finite-element method for calculating crankshaft deformation and bearing load of an engine. Proceedings of the institution of mechanical engineers part j-journal of engineering tribology, vol. 224, pp. 299-303.

Walczyk, W., Milenin, A., Pietrzyk, M. (2011). Computer Aided Design of New Forging Technology for Crank Shafts. Steel research international, vol. 82, pp. 187-194.

Zhao, Y., Cao, S.Q. (2013). Modal Analysis of Largene Crankshaft. Frontiers of manufacturing and design science III, pts 1 and 2, vol. 271-272, p. 1022-1026.

Date of submission of the article to the Editor: 07/2018

Date of acceptance of the article by the Editor: 09/2018 